\author{
Domenico D'Amico \\ Susanna Usai \\ Licia Grazzi \\ Massimo Leone \\ Andrea Rigamonti \\ Chiara Nespolo \\ Gennaro Bussone
}

\section{Disability and migraine: MIDAS}

D. D'Amico • S. Usai • L. Grazzi • M. Leone

A. Rigamonti • C. Nespolo • G. Bussone (凶)

C. Besta National Neurological Institute,

Via Celoria 11, I-20133 Milan, Italy

e-mail: bussone@istituto-besta.it

Tel.: +39-02-2394264

Fax: +39-02-70638067

\begin{abstract}
Migraine is an heterogeneous disorder. Most patients are disabled both in work and in non-work activities. Different instruments to assess migraine-related disability have been developed. Among these, the migraine disability assessment (MIDAS) questionnaire is the most studied. Population-based studies have shown that MIDAS is a simple but reliable and scientifically sound instrument. This instrument improves patient-physician communication. It can help healthcare professionals to understand migraine severity in individual patients, and rapidly assess treatment needs (screening instrument). Furthermore,
\end{abstract}

reduction in headache-related disability is a major goal of migraine treatment. Change in MIDAS score after treatment intervention may be a useful end point, both in everyday practice and in clinical trials. Ongoing studies will confirm the potential of MIDAS as a valid outcome measure.

Key words Migraine • Disability • Migraine disability assessment (MIDAS) questionnaire • Communication • Outcome measure

\section{Introduction}

Migraine is a typically episodic disorder, in that days with attacks are broken by periods without headache. However the attacks recur, and may be present throughout life; furthermore migraine is heterogeneous [1], and the intensity of the pain and associated manifestations vary in a given patient and between individuals [1-3]. In at least $70 \%$ of patients, the pain limits or impedes normal activities, consonant with "severe or moderate pain" being one of the diagnostic criteria for migraine [4]. Head movements and physical activity usually worsen the pain, so that patients tend to avoid brusque movements and, if they can, repair to bed. The phenomena associated with the pain also affect performance. The presence of nausea, photophobia, phonophobia
- in some cases, vomiting or diarrhoea, asthenia, shivering, blurred vision, absolute intolerance to sensory stimuli and the transitory neurologic phenomena of aura all contribute to adversely affect the physical and intellectual performance of patients.

\section{Disability and migraine}

Some patients have to stop work and go home if a migraine attack occurs; most who remain at work report reduced performance and efficiency. This results in increased workrelated insecurity and may have a negative influence on career [5, 6]. Activities outside work are also impaired [7]. Thirty percent of patients renounce family or social engage- 
ments because of their headache [8]. A patient's reduced receptiveness as a result of the headache may also have a negative effect on the relationship with the spouse, and may curtail sexual activity [9].

In view of these widespread but variable consequences of migraine, it is important to have instruments that can accurately assess migraine-related disability, i.e. the impact of migraine on patients' lives and activities [10].

\section{Instruments for assessing disability in migraine}

In 1994, Jacobson et al. [10] published their Henry Ford Hospital headache disability inventory (HDI) for assessing the self-perceived effects of headache. The HDI contains 25 questions grouped into emotional and functional domains. More recently, two additional headache questionnaires have been developed: the HIT-6, a questionnaire derived from the headache impact test (HIT-DynHA) available on the Internet [11], and the 24-hour headache disability questionnaire (Disq 24) [12], designed to quantify disability in three domains (family-social activities, work activities, and emotions-feelings) on a daily basis.

A series of disability instruments has been developed by the group of Lipton, Stewart, and Von Korff. These are the chronic pain index (CPI) [13], headache impact questionnaire (HIQ) [14], and migraine disability assessment (MIDAS) questionnaire [15]. The CPI classifies the pain of chronic pain syndromes (such as low back pain, facial pain, and headache) using a six-point scale: the score expresses both pain severity and functional limitations related to the pain. The principal advantage of this questionnaire is that scores predict unemployment and the need for medical assistance. The HIQ is a headache-specific instrument consisting of 16 questions about headache characteristics (intensity and frequency) and limitation of daily activities. Neither CPI nor HIQ are convenient to use in that the score represents an aggregate of measures and the system used to calculate it is relatively complex.

The MIDAS questionnaire is the most extensively studied instrument for assessing headache-related disability. It is particularly simple to use and it has been scientifically validated $[15,16]$.

\section{The MIDAS questionnaire}

The MIDAS questionnaire captures headache-related disability in all life domains over a 3-month period. This time interval was chosen by the authors to balance the accuracy of self-reported information with the clinical relevance of headache experience over time. It contains only seven questions and has simple and intuitive scoring rules. The first five questions investigate the influence of headache on three domains: questions 1 and 2 investigate paid work, enquiring as to the number of days off work due to headache and the number of days in which productivity was reduced by $50 \%$ or more; questions 3 and 4 ask the same questions about household work; question 5 enquires about missed days of recreational, social and family activities. Two additional questions (A and B) concern headache frequency (number of days with headache in the previous three months) and headache intensity (average pain intensity of headache attacks); they are not scored to calculate disability, but were included to provide the physician with clinical information. The total score is obtained by summing the scores (number of days affected) of the first five questions.

Population-based studies conducted in the USA and UK $[15,16]$ showed that MIDAS is a sound instrument with good test-retest reliability (Spearman's correlation coefficients, 0.78 and 0.77 , respectively). Internal consistency was also good (Cronbach's alpha greater than 0.7 ). The validity of MIDAS was evaluated by comparison with diary-based measures [17] in which patients complete a daily card, recording and scoring all activities and headache characteristics. The results indicate that the questionnaire provides a reasonably accurate measure of headache-related disability.

\section{Potential applications of the MIDAS questionnaire}

The simplicity of use and scientific validity of MIDAS suggest it is a useful tool for improving patient-physician communication, for patient screening and informing, and for evaluating treatment decisions. In fact, only a minority of migraine patients spontaneously report their headache-related functional impairment [18]. Similarly, most doctors are not aware of the real impact migraine has on their patients' lives and do not usually ask directly about the overall severity of the headache. The MIDAS questionnaire provides an opportunity for patients to indicate illness severity that is useful to the physician. MIDAS can therefore help healthcare professionals and primary care physicians to understand migraine severity in individual patients, to rapidly assess treatment needs and, in particular, to determine whether referral to a tertiary care structure is advisable. Reduction in headache-related disability is now considered a major goal of migraine treatment, as described in the recently published guidelines for the management of headaches developed by the US Headache Consortium [19].

Reduction in functional disability is also an important issue in clinical research. At present, the most important endpoint in clinical trials designed to assess the efficacy of 
acute medications for migraine is the headache response (relief of pain), usually after two hours [20]. It is becoming recognised that outcome measures expressing the patient's perspective are required: change in MIDAS score after treatment may be a useful endpoint in this respect. The Headache Centre of the C. Besta National Neurological Istitute is currently involved, as scientific coordinators, in a multicentric Italian study to assess these aspects. The study, called SIZE$\mathrm{M}$, is sponsored by Astra-Zeneca. The study recruited 327 patients from 35 centres and was designed to assess migraine disability using MIDAS at the moment of recruitment and again after six months of treatment with zolmitrip$\tan$ (Zomig Rapimelt). Data analysis, still ongoing, will indi- cate whether MIDAS is a valid and useful migraine outcome measure, i.e. whether it reflects the measured clinical improvement due to triptan administration.

In view of its utility in migraine screening, migraine outcome evaluation and patient-physician communication, MIDAS may have an important role in the co-ordination and assessment of healthcare initiatives for migraine management. Although migraine is a common condition and is increasingly recognised to adversely affect the personal, social and working lives of those who suffer from it, it is still underestimated by health policy-makers and physicians. Under-consultation, under-diagnosis and under-treatment mean that migraine is still, to a large extent, a hidden illness $[18,21]$.

\section{References}

1. Stewart WF, Schechter A, Lipton RB (1994) Migraine heterogeneity. Disability, pain intensity, and attack frequency and duration. Neurology 44[Suppl 4]:24-39

2. Henry P, Michel P, Brochet B, Dartigues JF, Tison S, Salamon R (1992) A nationwide survey of migraine in France: prevalence and clinical features in adults. Cephalalgia 12:229-237

3. Rasmussen BK, Olesen J (1992) Migraine with aura and without aura: an epidemiological study. Cephalalgia 12:221-228

4. Headache Classification Committee of the International Headache Society (1988) Classification and diagnostic criteria for headache disorders, cranial neuralgias and facial pain. Cephalalgia 8[Suppl 7]:1-96

5. Von Korff MR, Stewart WF, Simon DJ, Lipton RB (1998) Migraine and reduced work performance. A population-based diary study. Neurology 50:1741-1745

6. Stewart WF, Lipton RB, Simon D (1996) Work-related disability: results from the American migraine study. Cephalalgia 16(4):215

7. Pryse-Phillips W, Firdlay H, Tugwell P, Edmeads J, Murray TJ, Nelson RF (1992) Canadian population survey on the clinical, epidemiologic and societal impact of migraine and tension-type headache. Can J Neurol Sci 19:333-339
8. Smith R (1996) Impact of migraine on the family. Headache $36: 278$

9. National Academy of Sciences/Institute of Medicine (NAS/IOM) (1991) Disability in America: toward a national agenda for prevention. NAS Press, Washington DC

10. Jacobson GP, Ramadan NM, Aggarwal SK (1994) The Henry Ford Hospital headache disability inventory (HDI). Neurology 44:837-842

11. Kosinsky M, Bjorner JB, Dahlof C, Dowson A, Garber WH, Cady R, Ware J, Batenhorst A (2000) Development of HIT-6, a paper-based short form for measuring headache impact. Neurology 56[Suppl 3]:A139

12. Kwong JW, Putnam GD, Batenhorst AS (2000) Validation of a questionnaire that measures short-term headache disability. Neurology 56[Suppl 3]:A140

13. Von Korff MR, Ormel J, Keefe FJ, Dworkin SF (1992) Grading the severity of chronic pain. Pain 50:133-149

14. Stewart WF, Lipton RB, Simon D, Van Korff M, Liberman J (1998) Reliability of an illness severity measure for headache in a population sample of migraine sufferers. Cephalalgia 18:44-51

15. Stewart WF, Lipton RB, Kolodner K, Liberman J, Sawyer J (1999) Reliability of the migraine disability assessment score in a population sample of headache sufferers. Cephalalgia 19:107-114
16. Stewart WF, Lipton RB, Whyte J, Dowson A, Kolodner A, Liberman JN, Sawyer J (1999) An international study to assess reliability of the migraine disability assessment (MIDAS) score. Neurology 53:988-994

17. Stewart WF, Lipton RB, Kolodner A, Sawyer J, Lee C, Loiberman JN (2000) Validity of the migraine disability assessment (MIDAS) score in comparison to a diary-based measure in a population sample of migraine sufferers. Pain 88:42-52

18. Lipton RB, Amatniek JC, Ferrari MD, Gross M (1994) Migraine: identifying and removing barriers to care. Neurology 44[Suppl 4]:S63-S68

19. Silberstein SD for the US Headache Consortium (2000) Practice parameter: Evidence-based guidelines for migraine headache (an evidence-based review). Report of the Quality Standards Subcommittee of the American Academy of Neurology. Neurology 55:754-763

20. Pilgrim AJ (1992) Methodology of clinical trials of sumatriptan in migraine and cluster headache. Eur Neurol 32:177-184

21. Micieli G (1993) Suffering in silence. In: Edmeads J (ed) Migraine: a better future. Cambridge Medical, Worthing, pp 1-7 